\title{
VOLATILE ORGANIC COMPOUND PLUME DETECTION USING WAVELET ANALYSIS OF VIDEO
}

\author{
B. Uğur Töreyin, A. Enis Çetin \\ Dept. of Electrical and Electronics Engineering \\ Bilkent University, 06800, Ankara, Turkey
}

\begin{abstract}
A video based method to detect volatile organic compounds (VOC) leaking out of process equipments used in petrochemical refineries is developed. Leaking VOC plume from a damaged component causes edges present in image frames loose their sharpness. This leads to a decrease in the high frequency content of the image. The background of the scene is estimated and decrease of high frequency energy of the scene is monitored using the spatial wavelet transforms of the current and the background images. Plume regions in image frames are analyzed in low-band sub-images, as well. Image frames are compared with their corresponding lowband images. A maximum likelihood estimator (MLE) for adaptive threshold estimation is also developed in this paper.
\end{abstract}

Index Terms - volatile organic compounds, fugitive emission, wavelet transform, parameter estimation

\section{INTRODUCTION}

Petroleum refineries and organic chemical manufacturers periodically inspect leaks of volatile organic compounds (VOC) from equipment components such as valves, pumps, compressors, flanges, connectors, pump seals, etc [1]. Common practice for inspection is to utilize a portable flame ionization detector (FID) sniffing the seal around the components for possible leaks [2]. A single facility typically has hundreds of thousands of such components. A FID based monitoring approach turns out to be a tedious work with high labor cost even if the tests are carried out on a quarterly basis.

Several optical imaging based methods are proposed in the literature for VOC leak detection as a cost-effective alternative [3, 4]. In these approaches, infra-red (IR) cameras operating at a predetermined wavelength band with strong VOC absorptions are used for leak detection.

Video processing based VOC plume detection method is proposed in this paper. The method processes sequence of image frames captured by a visible-range camera. Although it is explicitly devised for visible-range cameras, techniques similar to the ones described in this paper can also be applied for image sequences captured by IR camera, as in [5].

There are different types of fugitive VOC emissions with varying plume characteristics. For example, diesel and propane have vapor similar to smoke coming out of a pile of burning wood whereas gasoline vapor is transparent and wavy. This results in visible, smoke like semi-transparent flickering plumes for propane and diesel. What is common in these VOC types is that the temperature of the VOC plume emitted from a leaking component drops during the initial expansion [1]. This causes a temperature difference between the VOC plume and the surrounding air. For gasoline, this temperature difference generates a fluctuation in the refractive index of the surrounding air causing blur and a net radiation difference by the gas absorption which can be observed with visible-range cameras.

Independent of the VOC type, plumes emitted from leaking components softens the edges in image frames. This characteristic property of VOC plumes is a good indicator of their existence in the range of the camera. It is well known that edges produce local extrema in wavelet subimages [6, 7]. Degradation of sharpness in the edges results in a decrease in the values of these extrema. However, these extrema values corresponding to edges do not completely disappear when there is VOC plume in the scene. In fact, they simply loose some of their strength but they still stay in their original locations, occluded partially by the (semi-) transparent plume. Therefore a decrease in wavelet extrema values or wavelet domain energy is an indicator of VOC plumes in the monitored area.

Regions with VOC plume are also analyzed in low-low subband images of wavelet transform. Energy of the image regions with plume is concentrated in low-low subband. Image regions with possible plume are compared with their corresponding low-low subband images.

Thresholds defined for high and low subband images are adaptively determined by a method using maximum likelihood estimation (MLE). This new approach is similar to the method described in [8]. In this way, the method adapts itself for various VOC types.

In Section 2, the adaptive background subtraction method to obtain a wavelet domain background image of the monitored scene is reviewed. Subband analysis based VOC plume detection method is described in Section 3. Threshold adaptation scheme is explained in Section 4. 
Experimental results and conclusions are presented in Sections 5 and 6.

\section{ADAPTIVE BACKGROUND SUBTRACTION IN WAVELET DOMAIN}

The first step of the VOC plume detection method is to detect changing regions in video. Background subtraction is a standard method for moving object detection in video. The current image of the video is subtracted from the estimated background image for segmenting out objects of interest in a scene. In [9], a computationally efficient method based on recursive background estimation method was proposed. In this paper, we use this method in wavelet domain to get an estimate of the background image.

Let $I_{n}(k, l)$ represent the intensity (brightness) value at pixel position $(k, l)$ in the $n$th frame. Estimated background intensity value at the same pixel position, $B_{n+l}(k, l)$ is calculated as follows:

$$
B_{n+1}(k, l)=\left\{\begin{array}{l}
a B_{n}(k, l)+(1-a) I_{n}(k, l),(k, l) \text { non - moving } \\
B_{n}(k, l),(k, l) \text { moving }
\end{array}\right.
$$

where $B_{n}(k, l)$ is the previous estimate of the background intensity value at the same pixel position. Initially, $B_{0}(k, l)$ is set to the first image frame $I_{0}(k, l)$. The update parameter $a$ is a positive real number where $0<a<1$. A pixel positioned at $(k, l)$ is assumed to be moving if the brightness values corresponding to it in image frame $I_{n}$ and image frame $I_{n-1}$ satisfy the following inequality:

$$
\left|I_{n}(k, l)-I_{n-l}(k, l)\right|>T_{n}(k, l)
$$

where $I_{n-l}(k, l)$ is the brightness value at pixel position $(k, l)$ in the (n-1)-st frame $I_{n-1}, T_{n}(k, l)$ is a threshold describing a statistically significant brightness change at pixel position $(k, l)$. This threshold is recursively updated for each pixel as follows:

$$
T_{n+1}(k, l)=\left\{\begin{array}{l}
a T_{n}(k, l)+(1-a)\left(c\left|I_{n}(k, l)-B_{n}(k, l)\right|\right), \\
(k, l) \text { non }- \text { moving } \\
T_{n}(k, l), \text { moving }
\end{array}\right.
$$

where $\mathrm{c}>1$ and $0<\mathrm{a}<1$, [9].

The wavelet transform of the background scene can be estimated from the wavelet coefficients of past image frames [10]. When there is no moving object in the scene, the wavelet transform of the background image is stationary as well. On the other hand, foreground objects and their wavelet coefficients change in time. Therefore equations (1)-(3) can be also implemented in the wavelet domain to estimate the wavelet transform of the background image as described in [10]. Let $D_{n}$ represent any one of the subband images of the background image $B_{n}$ at time instant $n$ : The subband image of the background $D_{n+1}$ at time instant $n+1$ is estimated from $D_{n}$ as follows:

$$
D_{n+1}(i, j)=\left\{\begin{array}{l}
a D_{n}(i, j)+(1-a) J_{n}(i, j),(i, j) \text { non - moving } \\
D_{n}(i, j),(i, j) \text { moving }
\end{array}\right.
$$

where $J_{n}$ is the corresponding subband image of the current observed image frame $I_{n}$. When the viewing range of the camera is observed for a while, the wavelet transform of the entire background can be estimated because moving regions and objects occupy only some parts of the scene in a typical image of a video and they disappear over time. Nonstationary wavelet coefficients over time correspond to the foreground of the scene and they contain motion information. In the proposed VOC plume detection algorithm, $D_{n}$ is estimated for the first level LL (low-low), HL (high-low), LH and HH subband images. These estimated background subband images are used in the subband based plume detection method.

Estimated subband image of the background is subtracted from the corresponding subband image of the current image to detect the moving wavelet coefficients and consequently moving objects as it is assumed that the regions different from the background are the moving regions. In other words, all of the wavelet coefficients satisfying the inequality

$$
\left|J_{n}(i, j)-D_{n}(i, j)\right|>T_{n}(i, j)
$$

are determined as moving regions.

\section{PLUME DETECTION}

As discussed in Sec. 1, fugitive VOC plumes soften the edges in image frames independent of the VOC type. It is necessary to analyze detected moving regions further to determine if the motion is due to plume or an ordinary moving object. Wavelet transform provides a convenient means of estimating blur in a given region because edges in the original image produce high amplitude wavelet coefficients and extrema in the wavelet domain $[6,7,11]$. When there is plume in a region wavelet extrema decrease. Therefore, (i) local wavelet energy decreases and (ii) individual wavelet coefficients corresponding to edges of objects in background whose values decrease over time should be determined to detect plume.

Let $J_{n, L H}, J_{n, H L}$ and $J_{n, H H}$ represent the horizontal, vertical and detail subbands of a single stage wavelet transform of the n-th image frame $I_{n}$, respectively. An indicator of the high frequency content of $I_{n}$ is estimated by

$$
\mathrm{E}_{\mathrm{h}}\left(\mathrm{I}_{\mathrm{n}}\right)=\sum_{i, j}\left|\mathrm{~J}_{\mathrm{n}, \mathrm{LH}}\right|+\sum_{i, j}\left|\mathbf{J}_{\mathrm{n}, \mathrm{HL}}\right|+\sum_{i, j}\left|\mathbf{J}_{\mathrm{n}, \mathrm{HH}}\right|
$$

Similarly for the background image $B_{n}$ :

$$
\mathrm{E}_{\mathrm{h}}\left(\mathrm{B}_{\mathrm{n}}\right)=\sum_{i, j}\left|\mathrm{D}_{\mathrm{n}, \mathrm{LH}}\right|+\sum_{i, j}\left|\mathrm{D}_{\mathrm{n}, \mathrm{HL}}\right|+\sum_{i, j}\left|\mathrm{D}_{\mathrm{n}, \mathrm{HH}}\right|
$$

Following inequality provides a condition for the existence of VOC plumes in the viewing range of the camera:

$$
\Delta_{1}(n)=\frac{\mathrm{E}_{\mathrm{h}}\left(\mathrm{I}_{\mathrm{n}}\right)}{\mathrm{E}_{\mathrm{h}}\left(\mathrm{B}_{\mathrm{n}}\right)}<T_{1}
$$

where the threshold $\mathrm{T}_{1}$ satisfies $0<\mathrm{T}_{1}<1$.

Candidate plume regions are determined by taking the intersection of moving regions and the regions in which a decrease in local wavelet energies occur according to (8). These candidate regions are further analyzed in low-low 
subband images. Most of the energy of the plume regions in image frames is concentrated in low-low subband. Hence, the difference between the average energies of plume regions in the current frame and its corresponding LL subband image is expected to be close to zero.

Let a single stage wavelet transform be used for subband analysis. Let a candidate plume region, $R s$ is determined in LL subband image, $J_{n L L}$ according to (5) and (8). Average energy of $R s$ is given as

$$
E_{R s, n}=\frac{1}{N} \sum_{(i, j) \in R s}\left|J_{n L L}(i, j)\right|^{2}
$$

where $N$ is the total number of pixels in $R s$. Average energy of the corresponding region, $R o$ in the original image $I_{n}$ is

$$
E_{R o, n}=\frac{1}{4 N} \sum_{(k, l) \in R o}\left|I_{n}(k, l)\right|^{2}
$$

Since the LL image is a quarter size of the original image, one needs to use a scaling factor of 4 to calculate the average energy of a pixel in (10). The candidate regions for which the difference between average energies is small are determined as plume regions:

$$
\Delta_{2}(\mathrm{n})=\left|\mathrm{E}_{\mathrm{Rs}, \mathrm{n}}-\mathrm{E}_{\mathrm{Ro}, \mathrm{n}}\right|<\mathrm{T}_{2}
$$

where $\mathrm{T}_{2}$ is a threshold.

Thresholds $T_{1}$ and $T_{2}$ are not fixed and adaptively determined by the following MLE based method.

\section{THRESHOLD ESTIMATION}

Thresholds $T_{1}$ and $T_{2}$ defined in Sec. 3 are adaptively estimated to account for various VOC types and changes in the lighting conditions. An MLE based threshold adaptation scheme is developed similar to the method in [8].

The clairvoyant MLE estimator for decision functions $\Delta_{1}(\mathrm{n})$ and $\Delta_{2}(\mathrm{n})$, defined in (8) and (11), is simply the sample mean estimator. Based on this estimator threshold values $T_{1}$ and $T_{2}$ can be easily determined. However the thresholds may not be robust to changing environmental conditions.

Let us consider the problem of estimating a threshold $\mathrm{T}$ in an adaptive manner from observed images. We assume that the threshold values vary according to the following expression for each image

$$
f[n]=T+w[n], n=0,1, \ldots, N-1
$$

where $w[n] \sim \mathrm{N}\left(0, \sigma^{2}\right)$ is zero-mean additive white Gaussian noise (AWGN) and $n$ is image frame number.

For each image frame, plume detection functions $\Delta(\mathrm{n})$ defines a binary image mask which is determined according to (8) and (11). One can also regard a binary mask as indicator variables defined by quantized observations $f[n]$ with respect to the threshold $\mathrm{T}$

$$
b(n)=1\{f[n] \in(\tau,+\infty)\}
$$

where $\tau$ is an initial parameter defining the mask $b(n)$.
Each $b(n)$ in (14) is a Bernoulli random variable with parameter

where

$$
q_{k}(T)=\operatorname{Pr}\{b(n)=1\}=F(\tau-T)
$$

$$
F(x)=1 /(\sqrt{2 \pi} \sigma) \int_{x}^{+\infty} \exp \left[-u^{2} / 2 \sigma^{2}\right] d u
$$

is the complementary cumulative distribution function of $w[n]$. In this case, the threshold is estimated in $N=10$ consecutive frames as follows $T=\tau-F^{-1}\left(\frac{1}{N} \sum_{n=0}^{N-1} b(n)\right)$, which can be obtained as in [8].

In this paper, we have two indicator functions $\Delta_{1}(\mathrm{n})$ and $\Delta_{2}(\mathrm{n})$, hence two separate thresholds, $\mathrm{T}_{1}$ and $\mathrm{T}_{2}$. We formulate and implement a more general approach than above which can be summarized in the following three steps:

1- Define a set of initial parameters $\tau=\left\{\tau_{u} \mid u=1,2\right\}$

2- Obtain binary observations $b_{u} ; \mathrm{u}=1,2$.

3- Find MLE for T.

Log-likelihood function is given as

$$
L(T)=\sum_{n=0}^{N-1} b_{u}(n) \ln \left(q_{u}(T)\right)+\left(1-b_{u}(n)\right) \ln \left(1-q_{u}(T)\right)
$$

from which the MLE of T can be defined as

$$
\hat{T}=\arg \max _{T}\{L(T)\}
$$

Since $\mathrm{T}$ in (17) cannot be determined in closed-form, Newton's algorithm is utilized based on the following iteration:

$$
\hat{T}^{(i+1)}=\hat{T}^{(i)}-\frac{\dot{L}\left(\hat{T}^{(i)}\right)}{\ddot{L}\left(\hat{T}^{(i)}\right)}
$$

where $\dot{L}(x)$ and $\ddot{L}(x)$ are the first and second derivatives of the log-likelihood function. Since the MLE problem (16), (17) is convex on T, the MLE in (18) is guaranteed to converge to the global optimum of $L(\mathrm{~T})$. These steps can be applied for both $\mathrm{T}_{1}$ and $\mathrm{T}_{2}$ separately.

\section{EXPERIMENTAL RESULTS}

The proposed method is implemented on a PC with an Intel Core Duo CPU $1.86 \mathrm{GHz}$ processor and tested for videos containing several types of VOC plumes including propane, gasoline and diesel. These clips also contain ordinary moving objects possible to trigger false alarms like shadows of swaying leaves on the surface of a pond.

The computational cost of the wavelet transform is low. The filterbank used in the implementation for single level wavelet decomposition of image frames have integer coefficient low and high pass Lagrange filters. Threshold updates are realized using 10 recent frames. 
Plume detection is achieved in real-time. The processing time per frame is less than $15 \mathrm{msec}$ for 320 by 240 pixel frames.

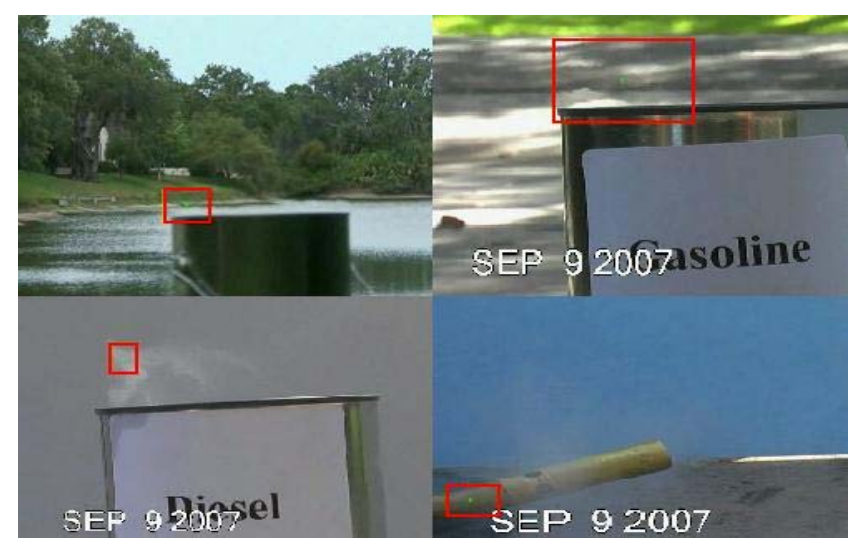

Figure 1. VOC plume detection results. Detected regions are marked with bounding rectangles. Image frames at the top row are gasoline plume, whereas images at the bottom are diesel and propane vapor.

Sample image frames with detected VOC plume regions are presented in Fig 1. Gasoline has transparent vapor whereas diesel and propane have semi-transparent regular smoke like plumes. Detection results for fixed and adaptive threshold methods for different VOC types are presented in Table 1. Threshold values are adjusted for gasoline type VOC plumes for the fixed threshold method in Table 1. Therefore, the detection performance for VOC plumes is decreased as well as the number of false positives is higher. No false alarms are issued for regular moving regions like shadows of swaying leaves when adaptive thresholds are used as shown in the last column.

Table 1: VOC plume detection results for adaptive and nonadaptive threshold implementations

\begin{tabular}{|c|c|c|c|c|c|}
\hline \multirow{2}{*}{ VOC type } & $\begin{array}{c}\text { Number of } \\
\text { frames } \\
\text { with } \\
\text { plume }\end{array}$ & \multicolumn{2}{|c|}{$\begin{array}{c}\text { Number of detected } \\
\text { frames }\end{array}$} & \multicolumn{2}{|c|}{$\begin{array}{c}\text { Number of false } \\
\text { positives }\end{array}$} \\
\cline { 3 - 6 } & $\begin{array}{c}\text { Fixed } \\
\text { thresh. }\end{array}$ & $\begin{array}{c}\text { Adapt. } \\
\text { thresh. }\end{array}$ & $\begin{array}{c}\text { Fixed } \\
\text { thresh. }\end{array}$ & $\begin{array}{c}\text { Adapt. } \\
\text { thresh. }\end{array}$ \\
\hline Gasoline & 1241 & 1088 & 1120 & 0 & 0 \\
\hline Diesel & 443 & 265 & 405 & 38 & 0 \\
\hline Propane & 310 & 120 & 288 & 14 & 0 \\
\hline
\end{tabular}

\section{CONCLUSIONS}

A VOC plume detection method based on wavelet analysis of video is developed. The method relies on comparing subband energies of image frames with those of background images. Thresholds defined for energy comparisons are updated by a novel MLE based adaptation scheme. The system proposes a cost effective alternative to flame ionization detectors which are currently in use to detect VOC leakages from damaged equipments in petrochemical refineries.

\section{ACKNOWLEDGEMENTS}

This work is supported in part by the Scientific and Technical Research Council of Turkey TUBITAK and European Commission 6th Framework Program with grant number FP6-507752 MUSCLE Network of Excellence Project. Authors are grateful to Prof. Giannakis for fruitful conversations on parameter estimation and to Mr. Dennis Akers for providing VOC vapor clips.

\section{REFERENCES}

[1] L. Zhou, and Y. Zeng, "Automatic alignment of infrared video frames for equipment leak detection," Anal.Chimica Acta, Elsevier, v. 584/1, pp. 223-227, 2007.

[2] U.S.EPA, "Protocol for Equipment Leak Emission Estimates," EPA-453/R-95-017, Nov.1995.

[3] ENVIRON, 2004. "Development of Emissions Factors and/or Correlation Equations for Gas Leak Detection, and the Development of an EPA Protocol for the Use of a Gas-imaging Device as an Alternative or Supplement to Current Leak Detection and Evaluation Methods," Fin.Rep.Texas Council on Env. Tech. and the Texas Comm. on Env. Quality, Oct., 2004.

[4] M.Lev-On, H.Taback, D.Epperson, J.Siegell, L.Gilmer, and K.Ritterf, "Methods for quantification of mass emissions from leaking process equipment when using optical imaging for leak detection," Environmental Progress,Wiley,v.25/1, pp.49-55, 2006.

[5] B.U.Toreyin, R.G.Cinbis, Y.Dedeoglu, and A.E.Cetin, "Fire Detection in Infrared Video Using Wavelet Analysis", SPIE, Optical Engineering, v. 46, June 2007.

[6] A.E.Cetin and R.Ansari, "Signal recovery from wavelet transform maxima,” IEEE Trans.on Sig.Proc.,v.42,pp.194-6,1994.

[7] S.Mallat, and S.Zhong, "Characterization of Signals from Multiscale Edges," IEEE Trans. on PAMI, v. 14/7, pp.710-732, July 1992.

[8] A.Ribeiro and G.B.Giannakis, "Bandwidth-constrained distributed estimation for wireless sensor networks - Part I: Gaussian case," IEEE Trans. on Signal Processing, v. 54, pp. 1131-1143, 2006.

[9] R.T.Collins, A.J.Lipton,T.Kanade,H.Fujiyoshi,D.Duggins, Y Tsin,D.Tolliver,N.Enomoto, O. Hasegawa, P. Burt, and L. Wixson, "A System for Video Surveillance and Monitoring: VSAM Final Report," Tech. report CMU-RI-TR-00-12,CMU,2000.

[10] B.U.Toreyin, A.E.Cetin, A.Aksay, and M. B. Akhan, "Moving Object Detection in Wavelet Compressed Video," Elsevier, Signal Proc.:Image Com., EURASIP, vol. 20, pp. 255264, 2005.

[11] A.Aksay, A.Temizel, and A.E.Cetin, "Camera Tamper Detection Using Wavelet Analysis For Video Surveillance," in Proc. IEEE Int. Conf. on Advanced Video and Signal based Surveillance, September, 2007. 